\title{
Fifty Years of Study of the Piezoelectric Properties of Macromolecular Structured Biological Materials*
}

\author{
M. KRYSZEWSKI \\ Department of Polymer Physics \\ Centre of Molecular and Macromolecular Studies \\ Polish Academy of Sciences, Sienkiewicza 112, 90-363 Łódź, Poland \\ (Received November 4, 2003)

\begin{abstract}
The piezoelectricity of biopolymers was discovered by E. Fukada for wood and bone in the fifties. This paper induced a number of studies on piezoelectric behaviour of bone collagen and tendon in wet and dry conditions as well as in many biological substances: polysaccharides, proteins, and biodegradable, optically active oriented films of poly(L-lactic acid). The implantation of this polymer induced the growth of bone, possibly because the ionic current caused by piezoelectric polarization stimulated the activity of bone cells. The phenomenon of bone growth has been discussed in terms of application of various substances, particularly modified collagen. The healing process of bone growth is still open both from the view of mechanism and biocompatibilities of materials used for this purpose. Fukada's group has a leading position in these studies. The fifty years of study of piezoelectricity in biomaterials resulted in many important observations and indication for further promising experimental and theoretical studies which will help to discover new ways and new materials for the tissue reconstruction.
\end{abstract}

PACS numbers: $83.80 . \mathrm{Lz}, 83.80 . \mathrm{Mc}, 89.20 . \mathrm{Bb}$

\section{Introduction}

Prehistoric man, at the beginning of his material, spiritual, and emotional culture, utilized macromolecular materials: plants, fruit and meat. These items were his nutrition. Wood and bone were the basic primary materials, which were

*Dedicated to Professor Eiichi Fukada who initiated studies on piezoelectricity in wood and bones. 
used as a source of light and heat, as well as for fighting. They were also the primary materials that men used to construct war tools used in the fight for survival. At first, these were sticks, picks, clubs, and the like used for fighting and for catching fish and game, as well as sometimes wild animals. The sharp and hard parts of the war tools were made from pieces of animal bones or teeth.

The wood and bones mentioned above belong to the macromolecular system-polymers of various chemical constitution and different morphological structures. It is a natural sequence of events that with the development of material culture throughout the centuries, human beings started being interested in more proficient uses of these natural biological substances, both in their development as objects of their material culture and in the expression of their spiritual feelings.

Up until now, the first microscopic observations have lead to some very important conclusions namely that those materials as well as all biological tissues have a hierarchical structure. They consist of macromolecular substances, which are organised into structures of different degrees of complexity. It seems worthwhile to note here that studies of natural macromolecules and particularly of synthetic polymers began at the end of the 19th century. In terms of the latter, it was easier to follow their development from synthetic chemistry up to more complicated physical structures in which polymers may exist. For the purposes of limiting the length of this paper, it is necessary to give up the discussion on the revolutionary development of the macromolecular concept and the evolution of the macromolecular system preparation as well as their further organisation into materials of various hierarchical structures, characteristic of different properties. The rapid progress in the chemistry and physics of synthetic polymeric systems was the basis for understanding the natural macromolecular systems. One can say that these two paths emerge due to many analogies and due to the use of the same modern techniques of the studies of biological and synthetic macromolecular systems. Still, we are far from the synthesis and fully understanding of the structure-property relationship of biologically important macromolecules such as DNA, DRA, protein enzymes, etc. The prospect, however, seems to be optimistic.

The principal aim of our discussion is the subject of the piezoelectric properties of wood and bones. We will briefly describe the skeleton structure of wood and bones and then go into some details of their piezoelectric properties. For the discussion of the latter problem, we need to present some necessary information on the basic physics of the piezoelectric effect.

The discussion of the piezoelectric properties of bones and their important implications will constitute the main part of this paper, followed by conclusions and some remarks on the prospects on the study in that field. The compactness of the text does not allow for listing all the necessary references, and so we wish only to present the principal works, particularly in the section devoted to a more general description of basic phenomena and in a greater number for substances and composites. 
There are three main classes of organic or organic/inorganic skeletal composites: wood, bone, and various shells, as well as other parts of living creatures. There is a considerable body of information collected during the last 50 years that indicates different aspects of the piezoelectric phenomenon of structural organic solids. For a concise presentation it is necessary to forego a more detailed discussion of polypeptides, except for collagen, polysaccharides, and cellulose $[1,2]$.

Collagen is the principal protein in the vertebrate's body. The main extracellular connective tissues consist of about $90 \%$ collagen in tendons and bones. Collagen may exist in different chemical constitutions and architectural organisations of collagen fibrils.

The triple helix is the specific structure, characteristic of collagen (tropocollagen). The primary collagen forms a "slow" left handed helix. Three of these helices coil around each other to give the collagen a triple helix. In order to create a fibre collagen macromolecules form a continuous structure which develops to allow for stress to be passed from one molecule to the next. This structure is very regular as shown by the banding patterns seen in electron microscope and X-ray diffraction.

Polymorphic fibrillar forms of collagen include four periodic fibrils, a set of obliquely banded fibrils and filament existing in a long spacing morphology. Immunology, immunogenicity, and antigenicity, all important properties of collagen, have to be left out of our present discussion.

In tendons and other structures, the collagen is associated with varying amounts of other proteins and acid mucopolisaccharides. Dry collagen is brittle and stiff with Young's modulus of $\sim 6 \mathrm{GPa}$. It is stiffer than hair and nearly as stiff as feather keratin. Wet collagen is not as stiff and an addition of water softens it progressively.

It is worthwhile to describe the organization of the tendon. Figure 1 clearly shows the hierarchical structure of this important unit of almost all living systems.

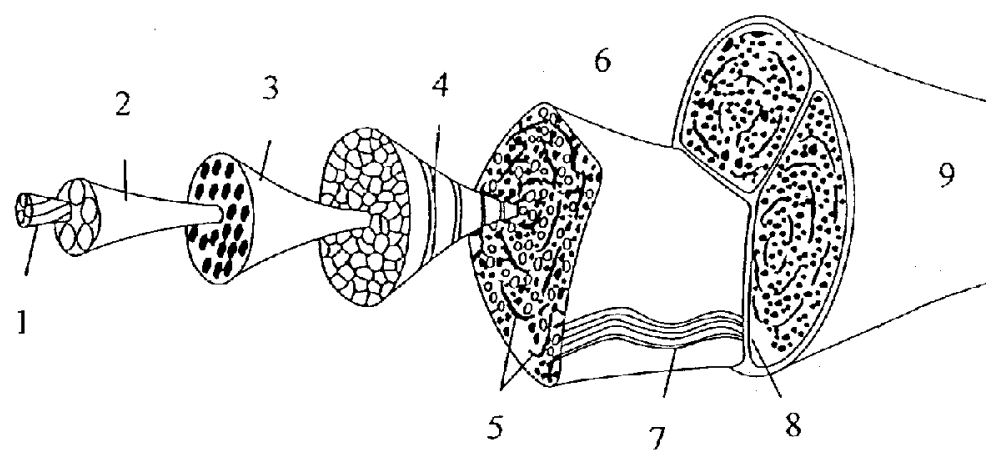

Fig. 1. The schematic organization of tendon (after [3]): 1 - tropocollagen, $2-$ microfibril, 3 - subfibril, 4 - fibril, 5 - fibroplasts, 6 - fascile, 7 - crimp structure, 8 - fascicular membrane, 9 - tendon. 
If the tendon is subjected to extreme stress, individual elements at different levels of the hierarchical structure may undergo a failure. However, the numerous elements depicted in Fig. 1 absorb the energy and protect the entire tendon from a final failure [3].

The tensile properties of tendon can be described in terms of the properties of tropocollagen (triple helix), which is composed of $70-80 \%$ dry collagen. The stress - a strain curve is typical of the low modulus material (low modulus plateau) attributed to the unfolding of crimp structures. The modulus of wet tendon in the step part of the curve is about $2 \mathrm{GPa}$. It is interesting to note that in ageing due to cross-linking the tendons strengthen, but fail at lower strains.

Cellulose belongs to the class of fibrous polysaccharides. The most important of these are chitin and cellulose. These two materials have a very similar primary structure. They probably polymerise just outside the cell membrane. An accepted hypothesis is that they could be formed by the immediate polymerisation into highly hydrogen-bonded elementary fibril with hydrogen bonds along or between the chains. Contrary to proteins in which $\beta$ sheets are possible, natural cellulose does not exhibit parallel and antiparallel forms. The cellulose elementary fibril is about $3.5 \mathrm{~nm}$ in diameter and contains $\sim 40$ molecules; in the case of chitin, the diameter of the fibril is about $2.8 \mathrm{~nm}$ and contains 20 molecules. Elementary fibrils up to $300 \mathrm{~nm}$ have been found in chitin in certain insects. Cellulose elementary fibrils may be combined into larger fibrils $20-25 \mathrm{~nm}$ in diameter. Both polymers are characteristic of high modulus, which in the case of cellulose has a maximum value of $14 \mathrm{GPa}$. A higher value was calculated from the crystalline parts of cellulose, assuming straightening of interchain hydrogen bonds. The actual stiffness of cellulose is lower since the crystallinity is lower and the chains are not strictly parallel.

In order to make a composite material stiffer, so that it can resist bending and compressive loads it is necessary to increase the shear stiffness of the matrix. It is also important that the stress is transferred from one fibre to another in the near neighbourhood. Usually, a new phase of ceramic material is introduced. Most commonly, calcium carbonate or phosphate salts are used in nature. Water, lipids, and salts as well as fillers may modify the stiffness of such a matrix. Fillers, cross-linkers, and mineral fillers can be easily incorporated, for example the cuticle of crustacea consists of about $50 \%$ calcite. About twenty molecules of chitin are combined to form microfibrils. The fibres transmit the stresses, and the proteins assemble the fibre into a matrix (no information exists about the arrangement of the matrix proteins). The orientation of one layer relative to the next can be varied, giving a plywood effect. One should also take discontinuities into consideration as these help prevent stress concentration. The mechanical properties can be changed to a high extent by thickness and water uptake.

Wood shows the same type of scaling and sophisticated architecture as that of animal materials. The organisation starts from the above-mentioned molecu- 


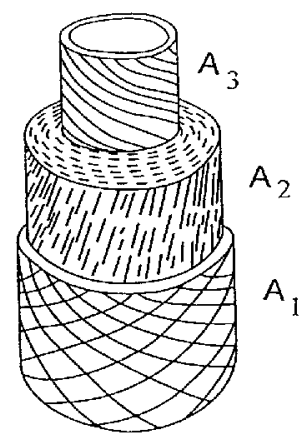

Fig. 2. Scheme of a typical wood cell: $A_{1}$ - primary wall, $A_{2}-$ secondary wall, $A_{3}$ - tertiary wall.

lar structure of elementary cellulose fibre and continues through to macroscopic columns of cells. A typical schematic wood cell is presented in Fig. 2. Cellulose is wound around three wall layers embedded in a matrix of lignin (it is a polymeric resin of polyphenol type). One can distinguish three layers: $A 1, A 2$, and $A 3$, which correspond to different orientations of fibrils. The most important is the layer $A 2$, which contributes up to $80 \%$ of the total thickness. It is the major load-bearing part of the wood cell. The helical arrangement of fibres results in a much higher structure energy as compared with that which is calculated from the frictional pullout measurements in synthetic fibres. We cannot go into detail regarding strength studies, but we can conclude that the $A 2$ layer creates a compromise between stiffness and toughness.

Bones are of major interest of us. They are made of crystallites of hydroxyapatite embedded tightly in collagen fibrils. The collagen is a template and it initiates the formation of crystallites. The first crystallite formed is precisely oriented with its crystallographic $c$-axis parallel to the long axis of fibre, and this orientation is retained as the calcification proceeds. The mineral crystallites in mature bone are $35 \mathrm{~nm}$ long ( $c$-axis). This is of the order of the gap region in the quarter stagger model of collagen. Not all the mineral in bone is crystalline; some amorphous regions exist as well, as can be seen by X-ray diffraction. The amount of amorphous material decreases with the increasing maturity of the creature. The detailed analysis of the packing of fibrils to make up the structure of bone is not complete as yet. One can however say that in the nanoscale, bone is a three-phase composite of hydroxyapatite platelet-reinforced collagen fibres in a macropolysaccharide matrix. Bone also has holes, which also are important biologically. A scheme of bone structure is presented in Fig. 3 .

The arrangement and packing of mineralised fibres control the direction and magnitude of stress which can be sustained, as well as the way in which the stress is transmitted through the bone. Generally, one can say that the fibrils are arranged in two ways: either in layers with a preferred direction, varying in a similar way to the layers of insect cuticle or as the secondary wall (cf. $A 2$ in Fig. 2) of wood 


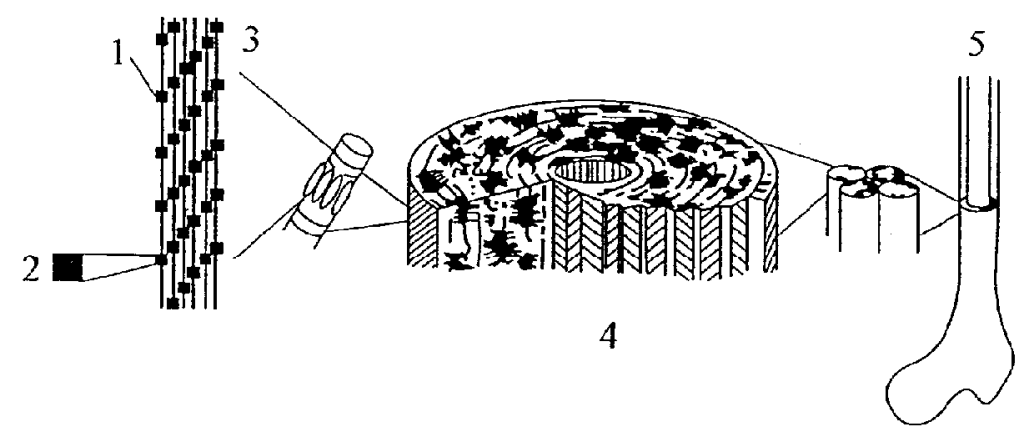

Fig. 3. Scheme of the structure of bone: 1 - tropocollagen, 2 - hydroxyapatite, 3 - microfibril, 4 - osteon, 5 - bone.

cell (lamellar bone) and/or in a more or less random way (woven bone). Much information about the arrangement of mineral phases can be obtained by studying fracture characteristic [2].

\section{Piezoelectricity - basic concepts}

Piezoelectric material characteristically develops an electric field (or voltage) when mechanically stressed along the appropriate direction, whereas an applied electric field $(E)$ produces a mechanical distortion in the material. These are called the "direct piezoelectric effect" and the "converse piezoelectric effect", respectively. The phenomenon of piezoelectricity was discovered by the brothers Pierre and Jacques Curie in 1880, in quartz, Rochelle salt, and some other single crystals, e.g. quartz ammonium dihydrogen phosphate $\mathrm{NH}_{4} \mathrm{H}_{2} \mathrm{PO}_{4}$ or $\mathrm{ZnS}$.

In accordance with our general aim we wish to mention the piezoelectric effects in composite materials. The first were studied heterogeneous piezoelectric ceramics such as polycrystalline barium titanate $\mathrm{Ba}\left(\mathrm{TiO}_{3}\right)$, a mineral that becomes piezoelectric after the application of a d.c. electric field. This process is called

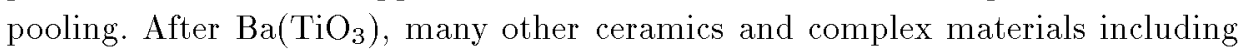
polymers were investigated with regard to their piezoelectric properties (see [4]).

There is an important difference between the converse piezoelectric effect and electrostriction, where the deformation is proportional to the square of the field. On the molecular level, piezoelectricity occurs when the elastic deformation of the body is accompanied by unequal vectorial displacements of centres of gravity of positive and negative charges (ions) leading to the polarization of the sample. The unequal vectorial displacements in the crystal require the lack of the crystallographic centre of symmetry.

A very strong correlation was observed between the symmetry of the crystal and the type of stresses, which cause the piezoelectric polarization. For the crystals of lower symmetry more types of stresses result in piezoelectric polarization. Each of the three components of the polarization $\Delta P_{i}$ is linearly related to each of the nine components of the stress tensor $\sigma_{j k}$. 


$$
\Delta P_{i}=d_{i j k} \sigma_{j k}
$$

where $d_{i j k}$ is the third-rank tensor having 27 components called piezoelectric coefficients. In the absence rotations, the stress tensor is symmetric, i.e. $\sigma_{j k}=\sigma_{k j}$. It has only six components thus the maximum number of piezoelectric coefficients is 18. For this reason it is convenient to represent the stress tensor as a six component vector $\sigma_{m}$ and consequently the piezoelectric coefficient as a second-rank tensor $d_{i m}$, were $m=1-6$ and

$$
\Delta P_{i}=d_{i m} \sigma_{m} \text {. }
$$

In a similar way for the converse piezoelectric effects each of the six components of the strain tensor $\varepsilon_{m}$ is connected to each of the three components of the electric field $E_{i}$, thus

$$
\varepsilon_{m}=d_{i m}^{c} E_{i},
$$

in which $d_{i m}^{c}$ is the converse piezoelectric coefficient. The stress and strain tensors are related to the elastic compliance tensor and to the elastic stiffness tensor

$$
\varepsilon_{m}=s_{m n} \sigma_{n}
$$

and so

$$
\sigma_{m}=c_{m n} \varepsilon_{n},
$$

where $c_{m n}$ (with $m, n=1-6$ ) is the elastic stiffness tensor and $s_{m n}$ - the elastic compliance tensor.

The brief description of values related to the piezoelectric effect aimed at introduction of the basic piezoelectric coefficients that will be used in the following text.

In the case of piezoelectric ceramics and polymer piezoelectric composites, the connectivity of the structure is the key feature. In a two-component system several connectivity patterns are possible. The most extensively investigated composites were PZT (lead-zirconate-titanate) composites ceramics or/and PLZT (lead-lanthanium-zirconate-titanate) composites [5].

Polymeric piezoelectric materials were studied later than a single crystal or ceramic piezoelectrics.

The best known polymer exhibiting piezoelectric behaviour is poly(vinylidene fluoride) $\left(\mathrm{PVF}_{2}\right)$ or (PVDF) which has the structure $\left(\mathrm{CF}_{2} \mathrm{CH}_{2}\right)_{n}[5,6]$. The piezoelectric polymer sample is obtained by orientation of dipole moments in the polymer by poling. The symmetry of the highly oriented $\mathrm{PVF}_{2}$ film reduces the number of possible piezoelectric coefficients. For uniaxially oriented films they are $d_{31}, d_{32}, d_{33}, d_{15}$, and $d_{24}$. For biaxially oriented films $d_{31}=d_{32}$ and $d_{15}=d_{24}$. The coefficient $d_{33}$ is very difficult to measure, so it is calculated from the hydrostatic piezoelectric coefficient

$$
d_{n}=d_{31}+d_{32}+d_{33}
$$

Stress-induced changes in polymer crystallinity significantly contribute to the piezoelectric coefficients. Due to the viscoelastic properties, the piezoelectric charge signals obtained from dynamic measurements depend on the frequency. 
It is worthwhile to note that these piezoelectric properties of organic polymer materials are small. They have been the inspiration for intense studies of piezoelectricity in synthetic polymers. It was demonstrated that substantial piezoelectric as well as pyroelectric effects can be observed when the films of PVDF are subjected to a strong d.c. field at elevated temperatures. Due to their flexibility and availability in the form of films of a large area, these materials have opened the door to possibilities for new applications and devices, some of which could not be realised with conventional crystalline piezoelectric materials. They can be applied as active elements in products ranging from infrared detector technology to loud-speakers. There is ample literature concerning the piezoelectricity of synthetic polymers, but we will cite only two very good reviews by Wada [7, 8], which perfectly describe the knowledge in the field. In addition to the PVDF related polymers, various other polymers have been studied in terms of their piezoand pyroelectric behaviour after poling (polyacrylonitryle, polyethylenterephtalate, polysulfone, polycarbonate, and $\mathrm{PVC}$, which is a noncrystalline polymer). It is piezoelectric when poled at a high temperature. Mopsik and Broadhurst [9] developed a dipole theory of the piezo- and pyroelectrocity of glassy polymer electrets, and obtained good agreement with the measured values. Work on polymeric piezoelectric materials continues in order to better understand their behaviour and to obtain simple and composite materials, as well as to find new applications and devices. In view of their piezoelectric behaviour, biological materials present a great interest. In the following section we wish to concentrate on wood and bone in particular $[10,11]$.

\section{Piezoelectric properties of wood and bone}

Studies of the piezoelectric properties of biological materials is a logical continuation of the investigation of Rochelle-salt crystals. These crystals are classical piezoelectric models of salts with organic components. The first organic skeletal structure, investigated in terms of viscoelastic properties and piezoelectric behaviour, was wood. This is indicated by Fukada in his excellent review article, which appeared in "Report on Progress in Polymer Physics in Japan" [12]. Studying the elastic constant and damping coefficient of wood over the acoustic frequency range, Fukada concluded interestingly that the high elastic constant and low damping capacity were an important criterion for wood used in instruments [13]. Another important property of wood is the specific change of viscoelasticity of this skeletal structure as a result of ageing. Fukada mentioned in 1950 [13] that the elastic constant of wood increases with time, reaching a maximum at 300 years. Subsequently it decreases with ageing, reaching a minimum value after 1300 years. This result was supported by X-ray diffraction investigations, which led to the conclusion that the degree of crystallinity of old wood increases for about 300 years and then decreases for up to 1300 years. After this long period of time, 
wood is almost $100 \%$ amorphous. In a natural environment the crystallisation of cellulose occurs slowly, but cellulose also degrades during the course of ageing.

Piezoelectricity is a property assigned to a number of organic crystals, hence Fukada, being very knowledgeable on the subject of the crystallinity of wood, began investigating the piezoelectric effects of this material. The detection of an electrical charge in wood was not an easy task due to the anisotropy of the skeletal structure of wood. It was necessary to cut out small samples of different orientations and to provide silver foil electrodes on the opposite faces, and then to detect the voltage created in the sample under pressure. Fukada discovered that very small voltage changes appeared when the wood cubes were compressed under a $45^{\circ}$ angle to the orientation of principal lamellae. This was the direct piezoelectric effect. In order to exclude the possibility that the electrical changes were induced by friction or contact potentials, studies were done to observe an inverse piezoelectricity in wood. It was natural to expect that the deformation of wood under pressure should be small, hence an interferometer microscope was used to measure the deformation under a high electric field. Unfortunately, these studies were unsuccessful due to the breakdown of the wood before deformation is large enough to be determined. Once again Rochell salt was used as a model and the application of alternative deformation made it possible (particularly at mechanical resonance) to detect the signal. The calculated values of piezoelectricity from direct and inverse piezoelectric effects agreed well. The piezoelectricity in wood originates from cellulose crystallites as well as from the anisotropic lamellar structure of this material. The cellulose fibres consist of crystallites in the oriented grain direction. There exist characteristics of triclinic symmetry and the eight components in the piezoelectric constant matrix are finite. Taking into consideration the nonpolar structure of wood, one can only expect there to be two components of the matrix. The shear piezoelectric constants are $-d_{14}=d_{25}$. The experimental results agreed with the theoretical predictions [14] and the magnitude of the shear piezoelectric constant of dry wood is $d_{14} \sim-0.1 \mathrm{pC} / \mathrm{N}$. It is small, and equals to one twentieth of that of quartz crystal. This is a remarkable discovery, which led to the conclusion that the piezoelectric effect should occur in various substances containing cellulose fibres. Since then, several other groups have investigated the piezoelectric effects of wood; also, piezoelectric signal appearance was used to evaluate the mechanical strength of lumber (an impulsive shock was applied at one end, and the piezoelectric signal was detected at a fixed distance). The piezoelectricity in bone has also a long tradition of research. In nineteen-fifties Fukada recognised soon that bones and wood are mechanically similar, both being uniaxially oriented structures. Wood consists of oriented cellulose crystallites, and in bones collagen plays an analogous role.

An investigation of piezoelectric effects in bovine and human bones confirmed that both direct and inverse piezoelectric effects can be detected in cubic samples of dried femur. The magnitude of the piezoelectric effect in bone was similar to 
that of wood. The polarization generated, both direct and inverse, piezoelectric effects were linear with two applied fields. The piezoelectric parameter of dry wood is $d=-0.2 \mathrm{pC} / \mathrm{N}$, which is twice that of wet wood. Fukada's theoretical considerations led to the confirmation of the anisotropy of this effect and to the derivation of the piezoelectric tensor of bones [15].

The studies conducted on the piezoelectric properties of bones initiated by Fukada have been undertaken by several groups. We mentioned above that bone consists of hydroxyapatite, which does not exhibit piezoelectricity; thus it was logically proposed, that tendons should be investigated as they are almost completely constituted of collagen.

Fukada and his collaborators [16] observed a large piezoelectric effect in dry tendons. Under shear, the piezoelectric coefficient of tendon was detected and piezoelectric constant was $d_{14}=-2.0 \mathrm{pC} / \mathrm{N}$, which is ten times that of bone. It was a surprise that tendon has a piezoelectric constant of the same value as quartz $\left(d_{11}=2.2 \mathrm{pC} / \mathrm{N}\right)$. In their initial experiments, Fukada et al. [11] applied shock or static pressure to the end of a femur clamped in a cantilever mode and measured the potential generated at the surface. In this experiment, usually termed the bending piezoelectricity experiment, the degree of polarisation is proportional to the derivative of stress. Quantitative bending piezoelectric experiments were carried out by Williams and Brigar [17].

In the early sixties the piezoelectric effect of wood and bones was a very new phenomenon. It is interesting to note that Fukada [12] demonstrated the piezoelectric effect in an ingenious way. He substituted a bone or a tendon element for a Rochelle salt crystal element in a commercial gramophone needle and successfully played records.

Further studies on piezoelectricity in tendon were related to bending effects. If pressure was directed parallel or perpendicular to the collagen axis, electrical polarisation was detected in the direction of orientation, but the piezoelectric constants $d_{33}$ and $d_{31}$ were two orders of magnitude smaller than that of $d_{14}$.

These studies on the piezoelectricity of bones and tendons initiated further investigations on the pyroelectric effect. The first results were published in 1969 [18], however they were not well checked and the supposed pyroelectricity was later attributed to the piezoelectric effect. Subsequent works on pyroelectricity have shown that both of these effects exist in many biological materials (not only in vertebrate skeletons). The work on piezoelectricity was initiated in the USA around 1960 and the results obtained there confirmed all of Fukada's group.

It is also interesting to note that the border between the collagen fibres and hydroxyapatite played an important role in the piezoelectricity of this system. It was interpreted in terms of the $p-n$ junction, a well-known concept in semiconductor physics. This topic will be discussed further when we describe heterogeneous piezoelectric systems. 
All the usual piezoelectrical materials are described by the matrix consisting of 18 piezoelectric constants. They represent the relations between the six components of mechanical stress and the three components of polarization. The collagen fibres in bone are nonpolar and uniaxial in symmetry, so only the shear piezoelectric constant, $d_{14}=-d_{25}$, has finite values. All other components are equal to zero.

The exact measurement of piezoelectricity in bones and tendons show, however, finite values of other components of piezoelectricity as well. This behaviour could be related to the heterogeneous structure of bone. There is another possible explanation of this effect. We can recall its other origin, it is the stress-induced potential which can be generated in bones.

Bone is a living material, which contains a considerable amount of water, and so it is natural to expect that the observed piezoelectric constant is a function of water content. The value of $d_{14}$ measured at $10 \mathrm{~Hz}$ is the highest in the dry state of bone and decreases with the water content being zero at about 30 polarization is neutralized by the ionic conduction of water.

The electromechanical effects in mineralised tissue are, as we have shown above, classical piezoelectric phenomena [15]. These results have enhanced studies of the piezoelectric current in bone in vitro. Lavine et al. [19] have shown in an ingenious way that the current can be easily monitored at any time by inserting a micro-ammeter into a circuit and the relation between the volume of the tissue (a slow rise in temperature) and the development of enhanced healing in the experiment as compared with control animals (rabbit) (see the references in [19]) could be detected.

The discovery of the electrical potential in bones focused the study in the direction of the streaming potential $\zeta$. Anderson and Ericson [20] published an interesting paper on the values of $\zeta$ in bones and concluded that the piezoelectric effect is only dependent upon applied strain and is not related to the $\mathrm{pH}$ of the surrounding solution. If any changes appear on the surface of strained fibre, they will be rapidly cancelled by the ions of opposite polarity absorbed from the solution, and thus the isoelectric point will change. In order to separate piezoelectric and streaming potential from piezoelectricity, Anderson and Ericson [20] investigated the pulsed voltage proving the existence of piezoelectric phenomena and streaming potential, both being dependent on the hydratation of collagen, the structural changes between dry and wet collagen have been taken into consideration, which is reflected in the symmetry of the collagen molecule. However, at small absorption of water, it is not possible for assumption of a particular symmetry group. The fact that the $\zeta$ value is equal to zero at the isoelectric point of the immersing fluid is valuable information because it demonstrates the role of the external ionic environment. Later on, intense investigations were devoted to a streaming potential. It was demonstrated that there is a distribution of electric potential across the section of bone being bent. This effect is ascribed to the streaming potential 
induced by the flow of fluid through canalicule, which are very fine tubes penetrating the structure of the bone. If a long bone is bent, the body fluids flow from the compressed regions to those which are expanded. The ionic strength of the fluid and the $\zeta$ potential at the surface of the canaliculi is determined in its sign and magnitude. The wet bone preserves its streaming potential in opposition to the piezoelectric effect.

The above-mentioned effects suggest that the external electrical field would affect bone and that bioelectrical stimulation would occur. This effect was indeed discovered and it is called the electrically mediated growth mechanism in living systems.

A characteristic feature of polymers in various fields is that they relax when the external field is switched off. Usually the mechanical properties of polymers are represented by complex elastic constants and in dynamic experiments a phase lag exists between the applied stress and the resulting strain. It is necessary to recall that the elastic constant represents the ratio of stress to strain and the angle between them. Both values are functions of frequency and temperature.

This piece of basic information on viscoelastic constants explains why the piezoelectric constants of polymers are complex values. Fukada [14] constructed a new experimental set up to measure dynamically the complex piezoelectric constant of polymer films. The first investigated sample was a thin plate of wood cut at an angle to fiber axis in order to show the piezoelectric effect. It was detected that the piezoelectric constant increases gradually as the temperature rises from $-150^{\circ} \mathrm{C}$ to $0^{\circ} \mathrm{C}$. The phase of polarization lagged the applied stress, which was obviously anticipated. However, when the temperature was above $0^{\circ} \mathrm{C}$, the piezoelectric constant decreased and the phase of polarization went, beyond the applied stress. When the piezoelectric constant is described, using imaginary numbers, by $d=d^{\prime}+\mathrm{i} d^{\prime \prime}$, the phase angle $\delta$ is given $\operatorname{tg} \delta=d^{\prime \prime} / d^{\prime}$. With an increasing temperature, $\delta$ was positive, whereas with a decrease in temperature, $\delta$ became negative. Such behaviour had never been observed in typical relations of elastic or dielectric constants. In the case of wood, at temperatures below $0^{\circ} \mathrm{C}$, the dipoles are oriented and this orientation is proportional to polarization. Above $0^{\circ} \mathrm{C}$, the ionic conductivity of water neutralises the dipolar polarization and it decreases with time [20]. Consequently, modified cellulose polymers such as cellulose diacetate and cellulose triacetate were the subjects of investigations in Fukada's laboratory, as was poly- $\boldsymbol{\gamma}$-glutamate [21] and poly- $\boldsymbol{\gamma}$-methyl-L-glutamate [22]. The latter is characteristic of a large piezoelectric effect and a retardation-relaxation effect connected with the side chains at a well-defined relaxation temperature. This polymer exhibits unusual piezoelectric characteristics. When the temperature increased from $-150^{\circ} \mathrm{C}$ to $150^{\circ} \mathrm{C}$ the piezoelectric constant $d=-d_{14}=d_{25}$ increased up to $0^{\circ} \mathrm{C}$, and then it decreased. The $\operatorname{tg} \delta$ also exhibited a similar behaviour (from positive to negative values). At about $0^{\circ} \mathrm{C}$, the elastic constants decreased and the dielectric constant $\varepsilon$ increased. 
This kind of behaviour was not expected but it could be explained by taking inhomogeneous materials into consideration. Date [23], Fukada's collaborator, has proposed a theory for the systems in which the piezoelectric inclusions of crystalline nature are uniformly dispersed in a non-piezoelectric amorphous medium. In this model, both the increase in $d$ and the increase in $\varepsilon$ are dependent on the interplay of amorphous and crystalline phases. This theory can be extended by introducing the concept of grain boundaries in the piezoelectric crystalline phase, which is particularly suitable for explaining the piezoelectric phenomena of the series of substituted poly(glutamates). With regard to these materials one has to consider the dipolar rotation in peptide groups in helical molecules, and that mechanical relaxation is connected with the thermal motion of side chains. The elastic constant of helices at grain boundaries is constant, but the motion is related to the chain (side chain) in the vicinity of helices [24].

Successful studies in the field of the piezoelectric behaviour of bones have inspired studies on a number of biopolymers. An excellent example is the shear piezoelectric effect in elongated and dried samples of blood cell walls. They consist of collagen, elastin, and muscle. Afterwords extensive work was carried out on separate dried myosin, actin, and muscle. At this point it is interesting to note the piezoelectric relaxation in deoxyribonucleate films below $0^{\circ} \mathrm{C}$ and the change of the piezoelectric constant with water content. Hydrated water induces a change in the helical structure of molecules - thus it was natural to expect significant effects on the piezoelectricity of DNA [25].

To sum up partially this section, we can say that polysaccharides, proteins, and deoxyribonucleic acids, the three basic types of biopolymers, have piezoelectric properties.

It is out of the scope of this paper to discuss in detail the piezoelectricity of other biological polymers, but it is necessary to say that piezoelectricity in biopolymers arises from atomic groups with asymmetric carbon atoms and polar groups such as CONH bonds, as well as from the specific uniaxial orientation of macromolecules.

These statements can be supported simply by indicating that oriented films of optically active poly(propylene oxide) exhibit distinct piezoelectric relaxation in the vicinity of glass transition temperature (about $-60^{\circ} \mathrm{C}$ ) and a piezoelectric constant $d_{14}=-0.1 \mathrm{pC} / \mathrm{N}$ at $100^{\circ} \mathrm{C}[25,26]$. Similar effects, but expressed more clearly, were observed in poly( $\beta$-hydroxybutylate) [27] and copolymers of hydroxybutyrate and hydroxyvalerate [28]. Poly(L-lactic acid) (PLLA) is of specific interest, as it exhibits piezoelectric relaxation at $T_{g}=85^{\circ} \mathrm{C}$ and $d_{14}=-10 \mathrm{pC} / \mathrm{N}$ in the room temperature range [29]. Poly(L-lactic acid) is a crystalline polymer, so the problem of its piezoelectric properties was discussed in terms of dispersion of crystallites in the amorphous phase, whereby the piezoelectric properties of the crystalline phase was found to be temperature independent [29]. These studies have shown that the magnitude of the shear piezoelectric constant in poly(L-lactic 
acid) is the highest ever observed in correlation with an unusual rotator power in the direction of the helical molecular axis.

The models for natural biopolymers are functional synthetic polymers usually investigated in the form of thin films. Some of them are prepared by a film casting technique and others by hot pressing, one such example is PVDF (which is hardly soluble). Their piezoelectric properties were mentioned, but there is a necessity to extend these studies in conjunction with a structural investigation using modern techniques, which provide intermediate access to the molecular level. The molecular beam vapour deposition allows for direct control of molecular structure (choice of proper support), thus making it possible to investigate the transformation during poling. There are now possibilities that more complex organic molecules can be vacuum deposited at low temperature, thus providing a material basis for direct correlation of piezoelectricity to structure. We will mention only two examples. One of the firsts is the paper published by Yoshida et al. [30] concerning the effect of electric field on the molecular orientation in evaporated ferroelectric vinylidene fluoride and trifluoroethylene (TrFE) copolymer films. It was shown that the electric field applied during evaporation process to obtain ultra thin films of (PVDT/TrFE) induces an uniaxial orientation of dipoles. The total reflexion X-diffractometry reveals that polar Form I crystallites with dipolar moment oriented normal to the substrate can be formed under high electric field (6 MV $/ \mathrm{cm}$ ) although the crystallinity of the films was low due to the pyrolysis of the sample during evaporation. Takahashi et al. [31] have shown that thin films of polyurea, less than $1 \mathrm{~mm}$, prepared by thermal vacuum deposition and polymerisation show an interesting piezoelectric effect. It seems on purpose to devote some more attention to this work. The stress-induced internal rotation of peptide bonds produces piezoelectric polarization. If the CONH group is ramplaced by urea bond HH COHN the value of dipole moment increases from 3.4 to 4.3 debye, thus the piezoelectric and pyroelectric properties are higher than those of polypeptides. The monomers: diisocyanate and diamine deposited on the surface of the substrate underwent addition polymerisation forming urea bonds. In this way the thin films of aromatic polyurea have been obtained (aromatic polyurea thin films cannot be obtained by spin-coating because of lack of solvent). Under higher electric field and of high temperature, urea dipoles are aligned in the some direction and large residual polarization, perpendicular to the film surface, is formed. The tensile piezoelectric constant was high as $10 \mathrm{pC} / \mathrm{N}$ and persisted at temperatures to $200^{\circ} \mathrm{C}$. Piezoelectricity of pooled films of polyurea not shear-induced as usually observed in elongated films of biopolymers. It is rather tensile stress-induced piezoelectricity which is observed for ferroelectrics ceramic substances.

Investigations of piezoelectricity in materials of biological origin are now in the stage of practical applications. The physiological importance of piezoelectricity in biopolymers has been discussed since the discovery of the phenomena discussed above. It was speculated that piezoelectric interaction may play a role in enzymatic 
catalysis, flagellar movement and in particular the sense of hearing and perception of pressure (see e.g. Lipinsky [32]). Most of these effects have been explained through the occurrence of selective changes in ion permeability. When an electrical field is applied to biomolecules an internal deformation of molecules occurs. It is a kind of movement which is similar to that of motion of animals and plants producing electric polarization.

A particular interest present the stress-induced potential of bone in vivo, which was already mentioned, e.g. the piezoelectric polarization of collagen in dry bone (when the water content is high, the piezoelectric polarization is neutralized by ionic current). It serves a purpose to consider once again the streaming potential which is caused by ionic flow through canaliculi, the small canals penetrating the structure of bone. The streaming potential is observed in wet bone.

These results created a great interest in the medical milieu. It is now generally accepted in orthopedics that the stress-generated potential is effective in new bone formation. The electric current is active in stimulation of the activity of bone cells thus it enhances the repair and growth of bone. The healing of pseudoarthrosis by electric stimulation is very important from the clinical view point (see, e.g. the book edited by Becker [33]). Many methods have been developed to insist the healing of fracture: direct current, alternating current, pulsing electromagnetic field, and ultrasonic field. Piezoelectric organic implants have been used too. Experiments carried (see [34]) have shown that the bone growth is enhanced by implantation of poly(L-lactic acid), tibia and fibula of a cat were cut and a rod of PLLA with diameter of $3 \mathrm{~mm}$ and lengths of $5 \mathrm{~cm}$ was inserted as an intramedullary pin. The X-ray taken the 8 th week after implantation indicated that a new bone is forming around the implanted pin if it consists of elongated PLLA. The increase in weight of the bone was correlated with the draw ration and the piezoelectric constant. It is interesting to note that in the case of oriented PLLA films, $0.2 \mathrm{~mm}$ thick, $6 \mathrm{~mm}$ wide, and $12 \mathrm{~mm}$ long, which were implanted between periosteum and muscle of rabbi tibia (the upper and lower ends of the film were fixed so that the elongation of the film produced piezoelectric polarization and thus induced ionic current) the most effective relative increment of new bone was detected after 6 weeks in the film oriented at $45^{\circ}$ vs. direction of orientation. It correlates well with the direction of maximum of piezoelectric effect. Since PLLA is degraded in the biological environment in about 6 months it seems to be a promising material as a bone inductive implant. It seems not necessary to continue in detail the discussion of medical use of organic piezoelectric structures in medicine particularly that the author was not involved in the cooperation with medical milieu using those materials. Why the electric current or electric field stimulates the activity of bone cells is an important question which is debated at present. Independently of piezoelectric activity which was rather extensively characterised above (see also a paper of Fukada [35]), another explanation consists of considering the enhancement 
of ionic transport across cell membrane caused by the electric field or mechanical stress (see e.g. Brighton and Pollack [36]).

Biomineralization is a complex phenomenon. Three principal stages are considered to occur during biomineralization: molecular preorganization, interfacial molecular recognition, and cellular processing as described by Mann [37]. An organized reaction environment such as lipid vesicle or protein cage or an extended protein-polysaccharide network is at first produced. This structure then provides a framework for mineralization and crystal nucleation is governed by electrostratic, structural, and stereochemical complementarity. Although the in vitro systems are highly complex, many elements can be investigated in artificial systems.

The piezoelectric synthetic or biological materials, when pooled, are characteristic of charge accumulation on the surfaces thus belong to the wide class of electrets. These materials create an external electrical field due to permanent electrical order of dipole moments of molecules or to accumulation of charges. Electret is in this respect similar to magnets which creates magnetic field. There are several methods to prepare electrets: e.g. polarization by heating in a strong electrical field (orientation of dipoles) and cooling down to stabilize the achieved orientation. Light and irradiation are often used to separate the charge and to fabricate electrets. Since the discovery of electrets by polarization of carnauba wax and resin with addition of bees wax by Eguchi in 1919 and obtaining photoelectret (sulfur electret) by Najakow (1938) the theory and fabrication as well as application of electrets were developed. Many original papers and books appeared concerning this interesting part of solid state physics in particular polymer and biopolymer physics. Let us recall as an example a very good book by Hilczer and Małecki [38] in which the readers may find a great deal of extensive information on the electrets.

We have introduced the term electrets only now because we wanted to follow the development of bioelectrets and their models according to the sequence of Fukada's group contributions. Evidently the studies on natural piezoelectric materials have adopted many experimental methods and concepts from the vast knowledge accumulated in the field of electrets, e.g. thermally stimulated current (TSC) or thermally stimulated discharge (TSD) technique which enables the spatial and energetical distribution of charges as well as distribution of charge density on electret surface. The measurements of depolarisation current, appearing by heating previously polarized several bone types, carried out by Mascarenhas [39], showed clearly that the charge stored due to polarization is high being of $10^{8} \mathrm{C} / \mathrm{cm}^{2}$ for a field of $10 \mathrm{kV} / \mathrm{cm}$ (saturation). These samples of bones were treated thermally and the role of collagen was suggested without considering the effect of denaturation.

The electret behaviour of collagen and blood vessel walls was also discussed by Mascarenhas [40]. He demonstrated that the blood vessels are able to polarization storage of $10^{-9} \mathrm{C} / \mathrm{cm}^{2}$. These results are evidently less concerning than the direct piezoelectric studies (due to the strong thermal treatment), however it is 
shown that the thermally stimulated current technique can be applied to biological material.

It seems worthwhile to note that the today TSC and TSD techniques together with a.c dielectric measurements are widely used for studies of materials of biological origin by many groups, e.g. of A. Konsta (Greece) and C. Lacabanne (France), leading to a better understanding of electrification phenomena. For the sake of the compactness of the text it is necessary to give up the details. Particularly interesting are the porous polymeric electrets which are of great importance because of unexpected large storage of charges. The porous electrets present today a major field of electret studies, see e.g. poly(propylene)cellular film which show, however, low thermal stability of piezoelectric coefficient. Porous poly(terrafloroethylene) films are an excellent non-polar electret material with a high space charge, outstanding thermal stability for both charge storage and mechanical properties [41]. It has a wide application for sensor and actuators. In biological piezoelectric material and their synthetic analogues like PLLA the piezoelectric activity is related to dipoles or ions and not so accumulated charges in pores by poling. However the structural inhomogeneity may play a certain role too.

At the end we wish to recall the recent experiments on anionic collagen for biomedical applications [42] and pyroelectric properties of a composite of anionic collagen: poly(PVDF/TrFE) [43]. This study describes the preparation of anionic collagen, characterization and determination of pyroelectric properties of anionic collagen and the mixtures with (PVDF/TrFE). It is an interesting work concerning natural and synthetic electro- and piezoelectric systems. It was shown that the increase in negative charges in collagen molecule significantly increases the pyroelectric coefficient and that the association with (PVDF/TrFE) produces materials with even higher coefficients without loss of tropocollagen secondary structure (1:1 composite). It is due to the hydrogen bonding between carboxyl and alcoholic hydroxyl groups of anionic collagen and fluorine atoms in the copolymer. Anionic collagen materials themselves are more attractive as active biomaterials. They show biocompatibility and tissue supporting ability. The anionic collagen membranes at $\mathrm{pH} 3.5$ submitted to fibroplast cell culturing demonstrated a high level of biocompatibility. Very inspiring are also results of bone growth support. Anionic collagen sponges with a net increase in negative charges of 36,53 , and 74 were compared with anionic collagen: a hydroxyapatite mixture. Defects filled with the material containing extra 53 negative charges are characteristic of bone neo-formation. The cavity was completely filled with a new bone tissue, with most of the fibers completely calcified. The bone formation was not accompanied to collagen matrices bioresorption, suggesting that they are used as a true support for a new bone formation. This material shows interesting properties for tissue engineering (see also the Proceedings of the 10th International Symposium of Electrets 1999 Delphi, Greece, for the information [43]). 


\section{Concluding remarks}

Many thousands of papers have been published on the piezoelectric, pyroelectric, and ferroelectric properties of single crystals, ceramics, and liquid crystals. They concerned physics, preparation, and application of such electroactive materials. Yet fewer than 100 papers have appeared related to these properties of biological materials. Piezoelectric and pyroelectric properties have been measured in a number of animal and/or plant tissues and the existence of piezoelectricity has also been documented. The important increase in interest in piezoelectric properties of macromolecular biological materials started with Fukada's discovery of piezoelectricity of wood and bones. Since the fifties of the last century some groups in the world followed the way of studies initiated by Fukada. Several biomaterials, both crystalline and amorphous, were investigated including polysaccharides, polypeptides and nucleic acids. A special significance had the studies on synthetic highly elongated films of poly(L-lactic acid) which was shown to be a piezoelectric active material. Bone collagen and particularly tendon exhibit shear piezoelectricity (in dry state) $-d_{14} \approx 2 \mathrm{pC} / \mathrm{N}$ which is comparable to this of quartz $d_{11} \approx 2.2 \mathrm{pC} / \mathrm{N}$. It seems justified to claim that piezoelectricity appears to be an almost universal property of biopolymers. Some of them have not been discussed in this paper, e.g. chitin, amylose, and keratin (wool, horn) but their general piezoelectric characteristics are similar to these of bone and wood. Water content in these materials is important because of the neutralization of a part of piezoelectric polarization by ionic current.

The most fascinating material among piezoelectric biological materials is bone. Bone as a complex hierarchical structure, which, despite great investigation, is still not well understood. The basic building blocks are extremely small plate shaped crystals of carbonate apatite. They are arranged in parallel layers within collagen network. The next hierarchical level are the mineral-filled collagen fibres which are organized into a 3 -dimensional structure that makes up a single layer or lamella of bone a few micron thick.

The investigation of apatite growth in a collagenous matrix belongs also to the topical questions because the calcification is studied mostly in model systems (in vitro) thus the transfer of obtained results to living systems is not obvious. Many authors however consider that the in vitro systems are a suitable mode to study the bone-biomaterial interactions.

Collagen is most abundant in animal tissue as a very long fibril with a characteristic axial periodic structure. How the fibrils are formed from their monomeric precursors is still the subject of studies. The collagen fibril formation is basically a self-assembly process but it is also sensitive to cell-mediated regulation particularly in young or healing tissues.

The studies on piezo- and pyroelectric properties of bone and collagen have contributed to these problems to some extent, particularly to research on the implant of bio-analogue polymers, e.g. poly(L-lactic acid). The very important 
parameter which is the necessary condition of their applicability is the biocompatibility.

We know, as yet that an absolute inert material does not exists, but biological response to any biomedical material must be kept in a certain range (we have introduced here the concept of biomedical materials which comprises well-known implants, like metals, ceramics, whereas biomaterials relate to systems created by nature).

The relationship between the physiological observations discussed here and piezoelectricity, pyroelectricity, and ferroelectricity are somehow speculative, except the bone healing activity. A great deal of further research will be necessary to demonstrate these correlations. The speculation can be an excellent initiative for investigations. The formed 19th century French physiologist, Claude Bernard in the introduction of one of his books wrote: "... in nature, what is absurd according to our theories, is not always impossible..." thus there is a hope that unexpected new results, theories, and applications of piezoelectric structured organic systems will be demonstrated. These studies are interdisciplinary and very challenging for both scientists and physicians.

\section{Acknowledgment}

This work was partially supported by the State Committee for Scientific Research, grant 4 T08E 04423 (2002-2005).

\section{References}

[1] Collagen: Biochemistry, Biotechnology and Biology, Ed. M. Nimmi, Vol. 3, CRC. Press Inc., Boca Raton (Fla) 1998.

[2] J.F.V. Vincent, Structural Biomaterials, Princeton Univ. Press, Princeton (NJ) 1990.

[3] Mechanical Properties of Biological Materials, Eds. J.F.V. Vincent, J.D. Currey, Cambridge University Press, Cambridge 1980.

[4] R.G. Kepler, R.A. Anderson, Advances in Physics 14, 1 (1992).

[5] Y. Xu, Ferroelectric Materials and their Applications, North-Holland, Amsterdam 1991.

[6] H. Kawai, Jpn. J. Appl. Phys. 8, 975 (1969).

[7] R. Hayakawa, Y. Wada, Adv. Polym. Sci. 11, 1 (1973).

[8] Y. Wada, in: Electronic Properties of Polymers, Eds. J. Mort, G. Pfister, John Wiley Son, New York 1982, p. 109.

[9] F.J. Mopsik, G. Broadhurst, J. Appl. Phys. 46, 4204 (1975).

[10] E. Fukada, M. Date, N. Hirai, Nature 211, 1079 (1966).

[11] E. Fukada, M. Tamura, I. Yamamoto, Rapport on the 6th Intern. Congress of Acoustics, 1968, D-69.

[12] E. Fukada, Rep. Prog. Polym. Phys. Jpn. 38, 1 (1995).

[13] E. Fukada, Nature 166, 772 (1950).

[14] E. Fukada, J. Phys. Soc. Jpn 10, 199 (1955). 
[15] E. Fukada, I. Yasuda, J. Phys. Soc. Jpn 12, 158 (1957).

[16] E. Fukada, I. Yasuda, Jpn. J. Appl. Phys. 3, 117 (1964).

[17] W.S. Williams, L. Brigar, Ann. New York Acad. Sci. 238, 127 (1979).

[18] S.B. Lang, Nature 224, 830 (1969).

[19] L.S. Lavine, J. Lustrin, M.H. Samos, Nature 224, 1172 (1969).

[20] J.C. Anderson, C. Ericson, Nature 227, 419 (1970).

[21] E. Fukada, M. Date, Nature 211, 1079 (1966).

[22] M. Date, S. Takashida, E. Fukada, J. Polym. Sci. A2 8, 61 (1970).

[23] M. Date, Polym. J. 8, 60 (1976).

[24] T. Furukawa, E. Fukada, J. Polym. Sci. Phys. Ed. 14, 1947 (1976).

[25] Y. Ando, E. Fukada, J. Polym. Sci. Phys., Ed. 14, 63 (1976).

[26] T. Furukawa, E. Fukada, Nature 221, 1235 (1969).

[27] Y. Ando, E. Fukada, J. Polym. Sci. Phys. Ed. 22, 1821 (1984).

[28] E. Fukada, Y. Ando, Int. J. Biol. Macromol. 8, 361 (1986).

[29] E. Fukada, Rep. Progr. Polym. Phys. Jpn. 34, 269 (1991).

[30] Y. Yoshida, T. Horiuchu, K. Matsushige, Jpn. J. Appl. Phys. 32, 1293 (1993).

[31] Y. Takahasi, S. Ukishima, M. Jijma, E. Fukada, Jpn. J. Appl. Phys. 28, 2245 (1989).

[32] Electronic Conduction and Mechanelectrical Transduction in Biological Materials Marcel Dekker, New York 1982.

[33] Mechanism of Growth Control, Ed. R.O. Becker, Charles C.Thomas Pub. Ltd., Springfield (IL) 1981.

[34] E. Fukada, Adv. Biophys. 6, 121 (1974).

[35] E. Fukada in: Medical Application of Piezoelectric Polymers, Eds. P.M. Galletti, D.E. Rossi, A.S. Reggi, Vol. 5, Gordon and Breach Science Pub., New York 1988, p. 15.

[36] Electromagnetism Medicine and Biology, Eds. C.T. Brighton, S.R. Pollack, San Francisco Press, San Francisco 1991.

[37] S. Mann, Nature 365, 499 (1993).

[38] B. Hilczer, J. Małecki, Electrets, PWN-Polish Scientific Publishers, Warszawa 1986.

[39] S. Mascarenhas, in: Electrets, Charge Storage and Transport in Dielectrics, Ed. M.M. Perlmann, Dielectric and Insulation Division, The Electrochemical Society Inc. Princeton, New Jersey 1973, p. 650.

[40] S. Mascarenhas, ibid, p. 657.

[41] W. Kuenstler, Z. Xia, T. Weinhold, A. Pucher, R. Gerard-Multhaupt, A. Appl. Phys. A 70, 5 (2000).

[42] A.M. Plepis, G. Goissis, D.K. Das-Gupta, Polymer Engineering and Science 36, 2932 (1996).

[43] Proc. of 10th Intern. Symposium on Electrets (ISE-10), Eds. A.A. Konsta, A. Vassilikou-Dova, K. Vartzeli-Nikaki, IEEE Dielectric and Electrical Insulation Society, Piscataway (NJ) 1999, p. 229 and p. 231. 